\title{
Perioperative course and accuracy of screw positioning in conventional, open robotic-guided and percutaneous robotic-guided, pedicle screw placement
}

\author{
Sven Rainer Kantelhardt • Ramon Martinez • \\ Stefan Baerwinkel • Ralf Burger • Alf Giese • \\ Veit Rohde
}

Received: 4 December 2010/ Accepted: 23 February 2011/Published online: 8 March 2011

(C) The Author(s) 2011. This article is published with open access at Springerlink.com

\begin{abstract}
Robotic-guided and percutaneous pedicle screw placement are emerging technologies. We here report a retrospective cohort analysis comparing conventional open to open robotic-guided and percutaneous robotic-guided pedicle screw placement. 112 patient records and CT scans were analyzed concerning the intraoperative and perioperative course. 35 patients underwent percutaneous, 20 open roboticguided and 57 open conventional pedicle screw placement. $94.5 \%$ of robot-assisted and $91.4 \%$ of conventionally placed screws were found to be accurate. Percutaneous robotic and open robotic-guided subgroups did not differ obviously. Average X-ray exposure per screw was $34 \mathrm{~s}$ in robotic-guided compared to $77 \mathrm{~s}$ in conventional cases. Subgroup analysis indicates that percutaneously operated patients required less opioids, had a shorter hospitalization and lower rate of adverse events in the perioperative period. The use of robotic guidance significantly increased accuracy of screw positioning while reducing the X-ray exposure. Patients seem to have a better perioperative course following percutaneous procedures.
\end{abstract}

Keywords Pedicle screw · Robotic-guided .

Percutaneous · Image guidance

\section{Introduction}

Pedicular screw placement is a standard procedure for spinal fusion in the lumbar and thoracic spine. In recent

This study contains parts of a doctoral thesis by S. Baerwinkel.

S. R. Kantelhardt $(\bowtie) \cdot$ R. Martinez $\cdot$ S. Baerwinkel •

R. Burger - A. Giese - V. Rohde

Department of Neurosurgery, Georg-August-University of

Göttingen, Robert-Koch-Strasse 40, 37075 Göttingen, Germany

e-mail: sven.kantelhardt@web.de years image guidance has increased the accuracy of pedicular screw placement [1-3]. Recent developments include a spinous process-mounted miniature robot, the SpineAssist $^{\text {TM }}$ (Mazor Robotics, Caesarea, Israel). Pechlivanis and colleagues [4] demonstrated the high accuracy of this robotic system. Nevertheless, with regard to the great variability of pedicle screw deviation rates given in literature (1-18\% [5-7]), it remains difficult for the surgeon to estimate the benefit of image guidance without a control group.

Likewise, a number of techniques for percutaneous pedicle screw placement have been introduced, mostly with favorable initial results [8-12]. However, no clinical study directly comparing the outcome of percutaneous and open midline approaches has yet been published in the English literature.

We here present a single institution retrospective analysis of perioperative results following lumbar and thoracic pedicle screw insertion using three different techniques.

\section{Materials and methods}

Patients

112 consecutive patients who underwent pedicular screw placement between January 2006 and August 2009 were analyzed regardless of the indication for spinal fusion.

\section{Surgery}

Pedicle screw placement was performed using three alternative techniques: patients received pedicle screws either using conventional 2-D fluoroscopic guidance (BV Endura, Philips, Hamburg, Germany) via a midline incision (termed 
"conventional"), or using an robotic-guided technique (SpineAssist ${ }^{\mathrm{TM}}$ Mazor Robotics, Caesarea, Israel). These robotic-guided procedures were either performed via a midline incision (open robotic-guided), or via a percutaneous approach. For percutaneous surgery separate $2 \mathrm{~cm}$ incisions for each screw and a 3-4 cm midline incision, to attach the robot to the spinous process by a clamp, were performed (percutaneous robotic-guided, Fig. 1).

In percutaneous cases additional PLIF- and TLIF-cage implantation and decompression of the spinal channel by laminotomy or laminectomy could be performed via the 3-4 cm midline incision when indicated (Fig. 2).

A pre-operative planning-CT was performed in all cases (16-slice MSCT-scanner, Aquilion ${ }^{\mathrm{TM}}$, Toshiba Medical Systems, Neuss, Germany). In robotic-guided cases 3-D reconstructions of this CT were used to plan the screw position using the SpineAssist ${ }^{\mathrm{TM}}$ planning-software. Registration of the SpineAssist ${ }^{\mathrm{TM}}$ robot was performed by matching the pre-operative CT scan with two intraoperative fluoroscopies (AP- and $60^{\circ}$ oblique images; BV Endura, Philips, Hamburg, Germany, see [6] for further details).

Six surgeons performed operations; all of them applied conventional and robotic-guided techniques. In 2006 all patients were operated conventionally, while robotic guidance was introduced in 2007 and implants for percutaneous surgery were introduced 2008. Following the introduction of the new techniques robotic-guided percutaneous surgery was preferred if the implants and instruments were available. The perioperative management, however, was not changed during this period.

\section{Implants}

Four types of pedicle screws were used: Tango ${ }^{\mathrm{TM}}$ (Ulrich medical, Ulm, Germany) and TRSH $3 \mathrm{D}^{\mathrm{TM}}$ (Medtronic,
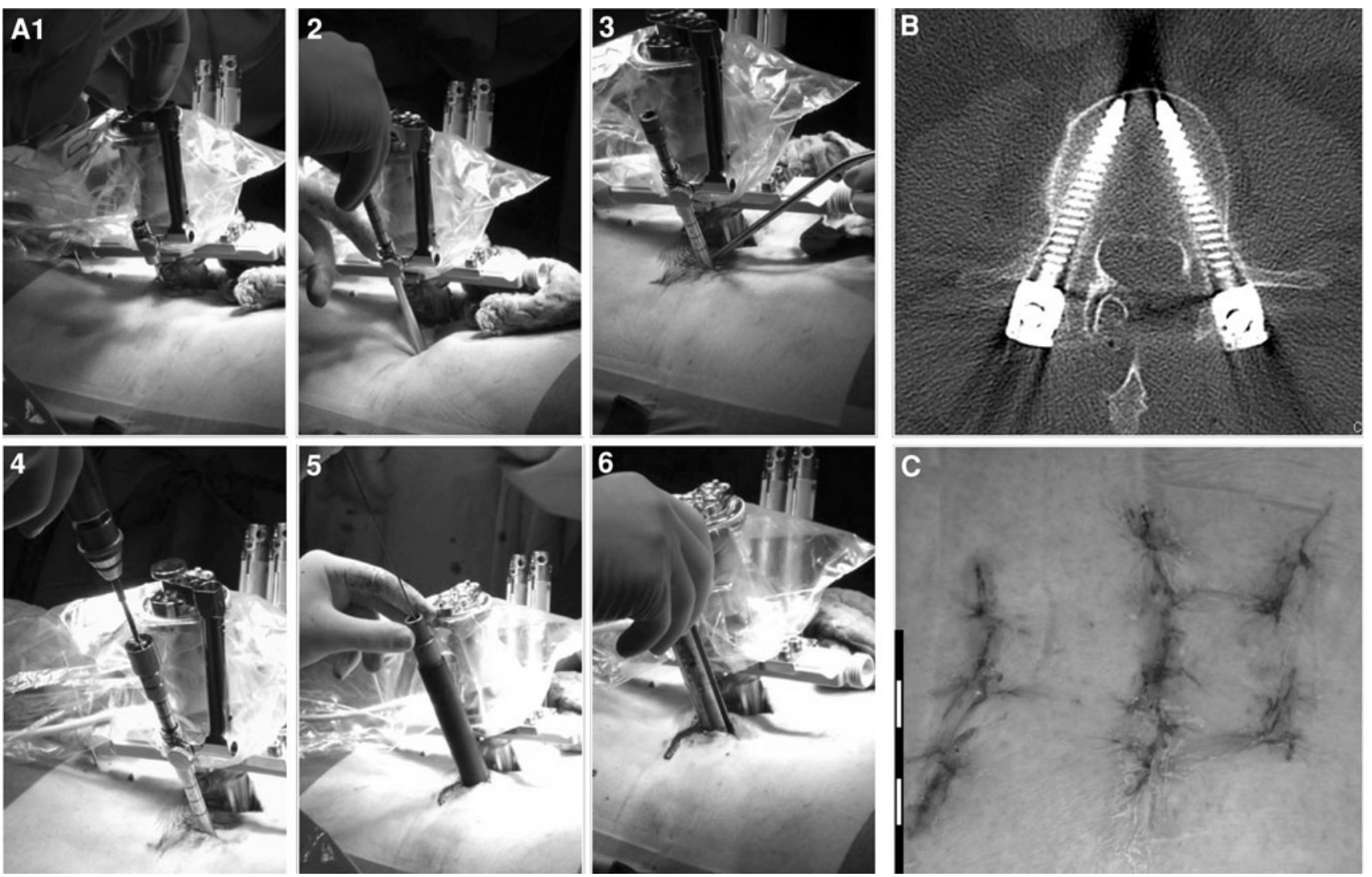

Fig. 1 Pictures a percutaneous robotic-guided procedure, as performed in 35 patients in this study. a1 shows the SpineAssist ${ }^{\mathrm{TM}}$ robot fixed to the spinous process by a clamp. The robot is already in the final position as planned by the surgeon on the planning-workstation. The arm which will guide drilling and guiding-wire insertion is being fixed to the robot. $\mathbf{2} 2$ shows placement of a guiding tube and marking of the skin at the planned entry point for pedicle screw placement. In $\mathbf{a} 3$ the skin has been incised at the marked spot and the guiding tube is inserted through the muscles to the entry point in the vertebral bone. a4 shows robotic-guided drilling of a transpedicular burr hole, a5 positioning of the guiding-wire and $\mathbf{a 6}$ percutaneous insertion of the screw. b shows a postoperative axial CT scan of the same screw and c displays the surgical wound postoperatively. The central incision was used for exposure of the spinous process and fixation of the robot and for placement of two PLIF cages. The four smaller incisions (two on each side) are the entry points of the percutaneously placed pedicle screws. The bar in the left lower corner of $\mathbf{c}$ is a $\mathrm{cm}$ scale 

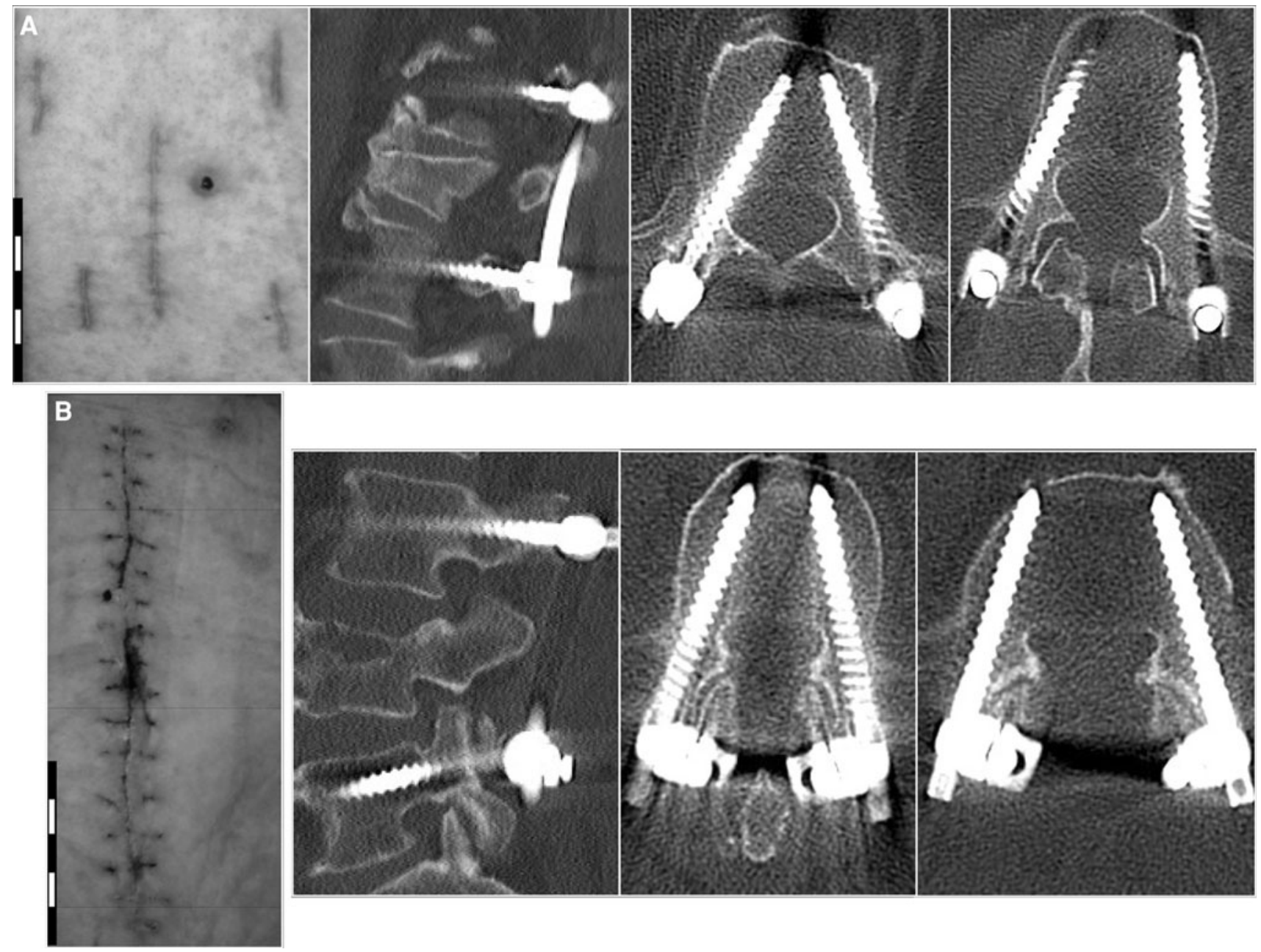

Fig. 2 a Shows the surgical scar (left image) and postoperative CT scan (one sagittal and two axial reconstructions) after a roboticguided percutaneous procedure (instrumentation from L2 to L4 because of osteoporotic L3 fracture). b Shows the same for a similar

case (L3-L5 because of osteoporotic L4 fracture) operated by conventional pedicle screw positioning via a midline incision. The bars in the left lower corners of the photographs are $\mathrm{cm}$ scales

Tolochenanz, Switzerland) in conventional procedures, Titan Expertise ${ }^{\mathrm{TM}}$ (Peter Brehm, Weisendorf, Germany) in robotic-guided and Icon $^{\mathrm{TM}}$ (Blackstone Medical, Laichingen, Germany) in percutaneous procedures. Screw diameter varied from 4.5 to $7.5 \mathrm{~mm}$.

\section{Assessment of screw position}

Screw position was assessed on CT scans obtained 1-4 days after surgery. Sagittal and axial reconstructions were analyzed by an investigator blinded to the insertion technique. The assessment was repeated 11 times using a millimeter scale; the relative numbers given in $\%$ represent the average values of these measurements. Screws entirely in the bone were graded 0 , grade 1 described an encroachment of the cortical bone, grade 2 a deviation of less than $3 \mathrm{~mm}$, grade 3 deviations from 3 to $6 \mathrm{~mm}$ and grade 4 for deviations of more than $6 \mathrm{~mm}$ (Fig. 3).

\section{Assessment of X-ray exposure}

Pre- and postoperative CT scans were performed routinely. The total intraoperative X-ray exposure was recorded and divided by the number of screws placed. For roboticguided cases this included the registration of the SpineAssist ${ }^{\mathrm{TM}}$.

\section{Collection of clinical data}

Intraoperative adverse events, operating time, postoperative hospitalization, postoperative administration of opioids, adverse events and reoperations were assessed. Analysis was based on routine-examinations directly postoperative, before discharge and about 12 weeks after surgery. All information was extracted from the patients' charts and CT scans. 
Fig. 3 Displays the system applied in this study for grading of pedicle screw deviations

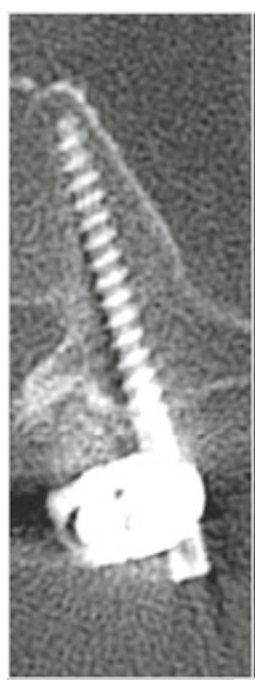

Deviation grade 0 entirely in the pedicular bone

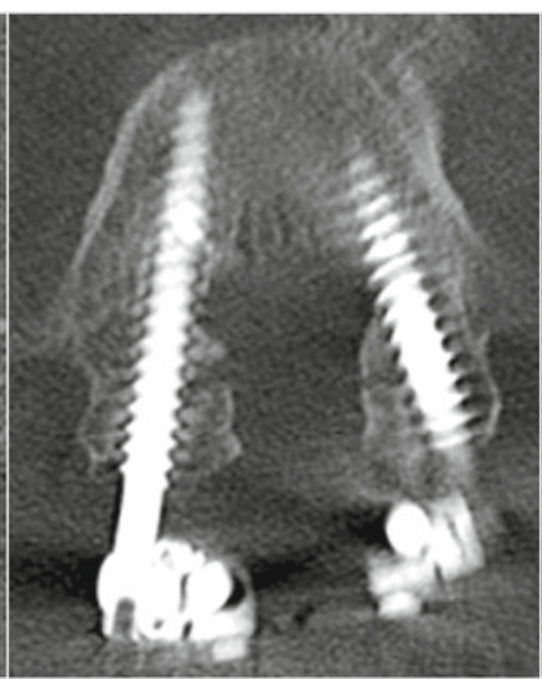

Deviation grade 1 Deviation grade
Encroachment of $2<3 \mathrm{~mm}$ the cortical bone

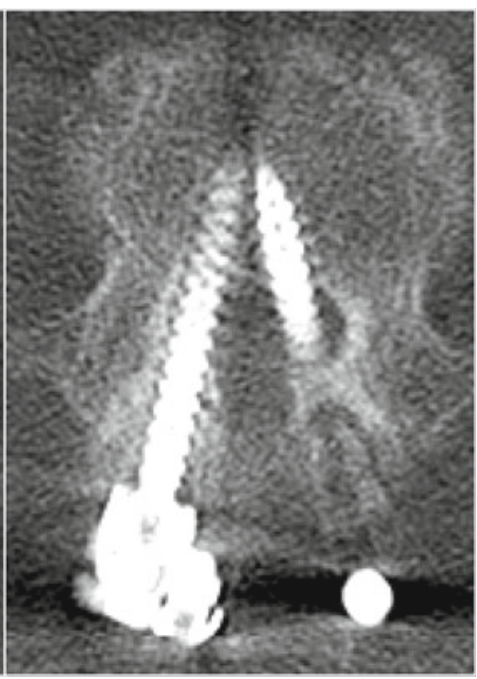

Deviation grade Deviation grade 3 3-6mm $4>6 \mathrm{~mm}$

\section{Exclusion from analysis}

Patients with spondylodiscitis were excluded from analysis hospitalization and infection rates, because their routine treatment included 3 weeks of i.v. antibiotics in hospital and they had infections prior to surgery.

Patients who were on opioids at hospital admittance were excluded from the analysis of postoperative opioid administration. Patients with incompletely documented data had likewise to be excluded from the concerned analysis (Table 1).

\section{Statistics}

Statistical significance was tested by student $t$ test when a Gaussian distribution was expected; in samples for which a normal distribution could not be assumed the Wilcoxon test was used instead. For unquantified data (nominal scale) the $\chi^{2}$ test was used. Significance level was set $\alpha=0.05$.

\section{Results}

Average patient age was 63.1 years, gender ratio $(\mathrm{m} / \mathrm{f})$ was 52/60. Average BMI was 26.8. These values did not significantly differ between the 3 (sub)groups (Table 2).

A total of 536 pedicle screws were placed in 112 patients. 286 screws in 57 patients were implanted conventionally and 250 (55 patients) in robotic-guided procedures. 94 of the robotic-guided procedure were performed via a midline incision (20 patients), while 156 screws (35 patients) underwent percutaneous procedures.
Table 1 The exact number of patients who have been included in the analysis of each parameter

\begin{tabular}{lllll}
\hline Number of patients analyzed & \multicolumn{2}{l}{ Robot-assisted procedures } & \multicolumn{2}{l}{$\begin{array}{l}\text { Conventional } \\
\text { procedures } \\
\text { Total }\end{array}$} \\
\cline { 2 - 5 } & Total & Percutaneous & Open & 57 \\
\hline Accuracy & 55 & 35 & 20 & 55 \\
Intraoperative X-ray exposure & 50 & 32 & 18 & 55 \\
Time for surgery & 54 & 35 & 19 & 27 \\
Opioid administration & 33 & 24 & 9 & \\
Adverse events & & & & 55 \\
$\quad$ Intraoperative & 43 & 33 & 10 & 28 \\
Infections & 37 & 27 & 10 & 50 \\
CSF fistula & 49 & 33 & 6 & 28 \\
Duration of hospitalization & 20 & 14 & 16 & 49 \\
Revision of screws & 49 & 33 & & \\
\hline
\end{tabular}


Table 2 An overview of patient characteristics for the analyzed groups and subgroups

\begin{tabular}{lllll}
\hline $\begin{array}{l}\text { Patient } \\
\text { characteristics }\end{array}$ & \multicolumn{2}{l}{ Robot-assisted procedures } & $\begin{array}{l}\text { Conventional } \\
\text { procedures } \\
\text { Total }\end{array}$ \\
\cline { 2 - 4 } & Total & Percutaneous & Open & \\
\hline $\begin{array}{l}\text { Number of patients } \\
\text { Age }\end{array}$ & 55 & 35 & 20 & 57 \\
$\quad$ Average & 62.8 & 62.7 & 62.9 & 63.4 \\
$\quad$ Min-max & $24-86$ & $27-82$ & $24-86$ & $16-85$ \\
$\begin{array}{l}\text { BMI } \\
\text { Average }\end{array}$ & 27.1 & 26.7 & 27.7 & 26.6 \\
$\quad \begin{array}{l}\text { Min-max } \\
\text { Gender ratio }\end{array}$ & $17-54$ & $17-54$ & $22-46$ & $19-36$ \\
m/f & & & & \\
\hline
\end{tabular}

No statistically significant differences concerning patient age, BMI and gender ratio could be found between the different groups and subgroups

Table 3 The relative frequency of deviation grades (0-4, compare Fig. 3) of pedicle screws for the different (sub-)groups (robotic-guided, percutaneous robotic-guided, open robotic-guided and conventional procedures)

\begin{tabular}{lrrrr}
\hline \multirow{2}{*}{ Grade } & \multicolumn{2}{l}{ Robot-assisted procedures $(\%)$} & Conventional procedures $(\%)$ \\
\cline { 2 - 3 } & Total & Percutaneous & Open & Total \\
\hline 0 & 90.5 & 90.6 & 90.2 & 87.8 \\
1 & 4.1 & 3.4 & 5.2 & 3.7 \\
2 & 4.3 & 4.9 & 3.3 & 5.0 \\
3 & 0.7 & 0.8 & 0.6 & 1.9 \\
4 & 0.4 & 0.3 & 0.7 & 1.6 \\
\hline
\end{tabular}

Accuracy of pedicle screw placement

$94.5 \%$ of robotic-guided and $91.5 \%$ of conventionally placed screws were graded 0 or 1 . Mild deviations (grade 2) were observed in $4.3 \%$ of robotic-guided and $5.0 \%$ of conventionally placed screws. Moderate (grade 3) and severe deviations (grade 4) were observed in 0.7 and $0.4 \%$ of robotic-guided and 1.9 and $1.6 \%$ of conventionally placed screws (Table 3). This was statistically significant regardless of whether grade 0 and grade 1 or exclusively grade 0 was rated as correct placement (Table 4). Results for percutaneous and open robotic-guided procedure did not differ obviously. Percutaneous procedures were significantly more accurate if only grade 0 was considered correct ( $p$ values, robotic-guided/conventional 0.00001; percutaneous/conventional 0.001 , open robot-guided/convent. 0.00001; percut./convent., 0.001), while open roboticguided procedures were superior if grade 0 and 1 were analyzed together (Table 3).
Table 4 The relative numbers of deviations for pedicle screws in different regions of the spine (thoracic, lumbar and sacral)

\begin{tabular}{ccccc}
\hline Grade & \multicolumn{2}{l}{ Robot-assisted procedures } & $\begin{array}{l}\text { Conventional } \\
\text { procedures } \\
\text { Total }\end{array}$ \\
\cline { 2 - 4 } & Total & Percutaneous & Open & \\
\hline \multicolumn{2}{l}{ Sacral region } & & & \\
0 & $90.6[28]$ & $91.8[20]$ & $87.5[8]$ & $88.8[26]$ \\
1 & 1.6 & 1.4 & 2.2 & 2.5 \\
2 & 4.2 & 4.1 & 4.5 & 3.5 \\
3 & 2.0 & 1.8 & 2.3 & 1.8 \\
4 & 1.6 & 0.9 & 3.4 & 3.5 \\
Lumbar region & & & \\
0 & $91.0[185]$ & $90.8[131]$ & $91.4[54]$ & $89.4[180]$ \\
1 & 3.9 & 3.5 & 5.1 & 4.3 \\
2 & 4.3 & 5.0 & 2.7 & 4.4 \\
3 & 0.5 & 0.6 & 0.3 & 1.1 \\
4 & 0.3 & 0.2 & 0.5 & 0.9 \\
Thoracic region & & & \\
0 & $87.6[36]$ & $77.3[4]$ & $88.9[32]$ & $85.7[80]$ \\
1 & 7.1 & 11.4 & 6.5 & 2.6 \\
2 & 4.3 & 6.8 & 4.0 & 7.0 \\
3 & 0.8 & 4.6 & 0.3 & 2.8 \\
4 & 0.3 & 0 & 0.3 & 0.9 \\
\hline
\end{tabular}

Values are expressed in percentage. The $\%$ values represent the average of 11 individual measurements, the real number of screws in each subgroup is given in square brackets

Intraoperative X-ray exposure

During robotic-guided procedures the average X-ray exposure was $34 \mathrm{~s}$ compared to $77 \mathrm{~s}$ in conventional cases. Average X-ray exposure for percutaneous robotic-guided screws was lower than $\mathrm{X}$-ray exposure during open roboticguided procedures ( $27 \mathrm{~s}$ compared to $43 \mathrm{~s}$ ). The differences between robot-assisted (both subgroups together and separately) and conventional procedures were statistically significant, the difference between percutaneous and open robot-assisted procedures was not statistically significant ( $p$ values, robotic-guided/convent. 0.0001; percut./convent. 0.001 , open robot-guided/convent. 0.023).

\section{Duration of surgery}

The duration of surgery documented in the patients' records always included all surgical steps from positioning to wound closure (including registration of the SpineAssist $^{\mathrm{TM}}$, PLIF- or TLIF-cages implantation, laminectomy, etc.). This operation time was divided by the number of screws placed. Average time per screw was found to be $59.1 \mathrm{~min}$ in robotic-guided $(57.0 \mathrm{~min}$ in percutaneous and $65.2 \mathrm{~min}$ in open robotic-guided procedures) and $52.9 \mathrm{~min}$ 
in conventional procedures. These differences were not statistically significant.

Postoperative administration of opioid analgesics

Postoperative routine included administration of NSAIDs. Novaminsulfone was added on request. When this regimen failed, opioids were added. $45.45 \%$ of the robotic-guided (37.5\% of percutaneous and $66.6 \%$ of open robotic-guided procedures), and $88.9 \%$ of conventionally operated patients who were not on opioids pre-operatively required postoperative administration of opioid analgesics (Table 5. Differences between robot-assisted and conventional and percutaneous robot-assisted and conventional (sub)groups were statistically significant $(p=0.0002$ and 0.004$)$.

\section{Adverse events}

Intraoperative adverse events (1 major hemorrhage, 6 dural tears) were observed in $4.7 \%$ of robotic-guided cases $(6.1 \%$ of the percutaneous and $0 \%$ of the open robotic-guided cases) and $9.1 \%$ of conventional procedures.

Postoperative cerebrospinal fluid fistulas were not observed following robotic-guided procedures $(0 \%)$, while $6.1 \%$ of the conventionally operated patients developed this complication. Postoperative infections occurred in $2.7 \%$ of robotic-guided $(0 \%$ after percutaneous and $10.0 \%$ after open robotic-guided procedures), while it was observed in $10.7 \%$ of the conventional procedures (Table 5). Statistical analysis showed significance between robot-assisted and conventional procedures for intraoperative adverse events only ( $p=0.04)$.

\section{Postoperative hospitalization}

Routinely patients were scheduled to remain ten postoperative days in hospital. Average hospitalization was
10.6 days following robotic-guided fusion (10.1 days for percutaneous, 11.6 days for open robotic-guided procedures) and 14.6 days following conventional procedures (Table 5). Differences between the robot-assisted and conventional and percutaneously and conventional (sub)groups were statistically significant ( $p$ values, robot-gui$\mathrm{ded} /$ convent. 0.009; percut./convent. 0.012).

\section{Revision surgery}

Revision of misplaced screws was performed in 8 cases; $1.0 \%$ robotic guided $(0.3 \%$ percutaneous and $6.3 \%$ open robotic-guided) and $12.2 \%$ conventional procedures (Table 5). Secondary sutures (mostly under local anesthesia on the ward) had to be placed because of wound healing disorders/infections in 10 cases $(0.6 \%$ percutaneous procedures, $12.6 \%$ open robotic-guided procedures and $12.2 \%$ conventional procedures). 1 case of dislocated PLIF cage had to be revised (open robotic-guided). No statistical significances were found.

\section{Discussion}

Perioperative clinical outcome and accuracy of pedicle screw placement in cohorts of robotic-guided (open and percutaneous) and conventionally placed pedicle screws were assessed. We focused on intra- and perioperative outcome, because the advantage of image guidance should show during surgery, respectively, on a postoperative CT scan, while the effect of a minimally invasive approach will be strongest during wound healing. Long-term results (fusion rate, etc.) were not included as it can be expected that these will be similar for all pedicle screws regardless of the surgical approach applied.
Table 5 The relative and absolute numbers of patients who required new opioid analgesics after surgery, the rate of adverse events (for intraoperative adverse events, wound healing disorders, postoperative infections and cerebrospinal fluid fistulas) the average duration of postoperative hospitalization and the rate of secondary surgical interventions

\begin{tabular}{|c|c|c|c|c|}
\hline \multirow[t]{2}{*}{ Grade } & \multicolumn{3}{|c|}{ Robot-assisted procedures } & \multirow{2}{*}{$\begin{array}{l}\text { Conventional procedures } \\
\text { Total }\end{array}$} \\
\hline & Total & Percutaneous & Open & \\
\hline Opioid administration & $45.5[33]$ & $37.5[24]$ & $66.6[9]$ & 88.9 [27] \\
\hline \multicolumn{5}{|l|}{ Adverse events } \\
\hline Intraoperative & $4.7[43]$ & $6.1[33]$ & $0[10]$ & $9.1[55]$ \\
\hline Wound healing & $13.5[37]$ & $11.1[27]$ & $20.0[10]$ & $21.4[37]$ \\
\hline Infections & $2.7[37]$ & $0.0[27]$ & $10.0[10]$ & $10.7[38]$ \\
\hline Cerebrospinal fluid fistula & $0[49]$ & $0[33]$ & $0[16]$ & $6.1[50]$ \\
\hline Postoperative hospitalization (days) & $10.6[20]$ & $10.1[14]$ & $11.6[6]$ & $14.6[28]$ \\
\hline Repositioning of screws & 1.0 [49] & $0.3[33]$ & $6.3[16]$ & $12.2[49]$ \\
\hline
\end{tabular}

Values are expressed in percentage and values in square brackets indicate the number of patients analysed 
Assessment of accuracy of screw positions

Assessment of pedicle screw position in replicate determinations by a single investigator blinded to the insertion technique was performed in order to minimize the effect of investigator-dependent errors. Assessment was based on the evaluation scale proposed by Wiesner [8] and Schizas [3].

Many clinicians accept deviations up to 2 or $3 \mathrm{~mm}$ because minor deviations rarely become symptomatic. Lonstein et al. [5] report in their meta-analysis of 4,790 screws of $5.1 \%$ screws breaching the cortical bone. No more than $0.2 \%$ of these caused neurological symptoms. Nevertheless, from time to time surgeons will be confronted with remaining/new-onset symptoms in the presence of a minor screw deviation and face the dilemma whether to reoperate or not. Therefore, we here recorded all minor and major deviations.

Image guidance

Following the literature conventional screw placement results in a deviation rate of $1-18 \%$ [5-7], while other authors described a significant reduction of this deviation rate when image guidance is applied (2.7-3.9\% [13-15]).

In percutaneous procedures deviation rates tend to be higher (between 6.6 and $80 \%[2,3,12,16]$ ) and image guidance has likewise been shown to reduce this [16].

Presently, a great variety of systems offering spinal image guidance are under research, clinical evaluation or already in routine clinical use. Among the proposed techniques are electromagnetic navigation [17], intraoperative 3-D fluoroscopy and CT navigation [16, 18], and percutaneous reference frames [19] and robotic-guided surgery [4].

\section{Accuracy rates}

Pechlivanis and colleagues [4] report in their recent study using SpineAssist ${ }^{\mathrm{TM}}$ of $91.7 \%$ of screws entirely within the bone, while further $6.8 \%$ of the screws showed deviations of less than $2 \mathrm{~mm}$. This corresponds to our results $(94.6 \%$ grade 0 and 1 and $4.3 \%$ grade 2 ).

The accuracy of conventionally placed pedicle screws in our study was $91.4 \%$ grade 0 or 1 and further $5.0 \%$ grade 2 . This corresponds to given in literature [5-7].

In accordance with the literature [16], in our study the accuracy of image-guided pedicle screw placement was significantly higher than in conventional procedures (about $3.1 \%)$. In robotic-guided procedures the surgical approach did have any obvious influence on the accuracy of screw positions. This contrasts with the situation in conventional procedures, as discussed above [2, 3, 16].
X-ray exposure

Intraoperative X-ray exposure was significantly reduced by application of robotic guidance. Pre- and postoperative CT scans were performed routinely, so that the SpineAssist ${ }^{\mathrm{TM}}$ planning-CT did not cause additional X-ray exposure. Furthermore, the preoperative planning-CT did not affect the surgical team which, in contrast to the patient, faces the intraoperative radiation frequently.

Interestingly, we observed a decrease of intraoperative $\mathrm{X}$-ray doses during percutaneous robotic-guided procedures compared to open robotic-guided procedures. The best explanation is, however, our learning curve with the SpineAssist $^{\mathrm{TM}}$. The first robotic-guided procedures in 2007 were open robotic-guided and the decreased radiation during percutaneous procedures might reflect a growing confidence in the SpineAssist $^{\mathrm{TM}}$ in 2008.

\section{Clinical parameters}

Duration of postoperative hospitalization, postoperative opioid administration (indicator of severe pain), infection rate and rate of screw revisions were better in the roboticguided group. However, the percutaneous subgroup did still better, while the open robotic-guided subgroup resembled more the conventional cases. This indicates that the perioperative clinical course depends less on the application of image guidance than on the surgical approach (percutaneous versus open).

\section{Percutaneous pedicle screw placement}

In literature many authors report good clinical results following percutaneous pedicle screw placement for various indications (trauma [9], spondylodiscitis [20] and spondylolisthesis [10, 11]). Accordingly, several studies measuring serum creatine kinase levels after surgery indicated less muscular trauma in percutaneous operations $[21,22]$. Cadaver and animal studies comparing percutaneous and open midline approaches supported these findings [23, 24].

However, studies directly comparing clinical outcome after percutaneous and open pedicle screw placement in humans are not yet published in the English literature. Grass and colleagues report in a German journal "Der Unfallchirurg" of 57 patients with thoracolumbar fractures. EMG examination proved greater trauma in open procedures and blood loss was significantly higher. Two of the 35 patients operated percutaneously needed a revision. However, the study focuses on intraoperative issues and accuracy of screw placement, further clinical data is not given in this interesting article [9]. Another study, which appeared in the Chinese language journal "Zhonghua Wai 
Ke Za Zhi" reports of a prospective study in 60 patients with thoracolumbar burst fractures, who had been treated with either percutaneous or open pedicular screw placement [25]. The authors conclude that "pedicle screw fixation for thoracolumbar fracture has the advantage of less trauma, quicker recovery and better esthetic outcomes, however, it has the same results with the traditional open produce after 2 years of surgery". Our study likewise indicates that clinical outcome following percutaneous procedures might be superior to that following an open midline approach.

\section{Shortcomings of the study}

The underlying pathologies were heterogeneous. Posterior stabilization with pedicle screws and rod systems was frequently combined with other surgical procedures. Therefore, the assessed parameters were not exclusively related to pedicle screw implantation, but may partly reflect additional surgical procedures. Some cases had to be excluded from the analysis of one or several parameters because of the underlying disease. This influences the quality of our data and the conclusions derived from it.

Besides this study spans from 2006 to 2009 and the robot-assisted (percutaneous) subgroup represents the most recently operated cohort. Patients were operated by six different surgeons and the data includes all their learning curves with percutaneous approaches, the SpineAssist ${ }^{\mathrm{TM}}$ and spinal fusion surgery in general. Additionally, no randomization was performed, so that an entry bias might further confound this study.

\section{Conclusions}

Robotic-guided pedicle screw implantation resulted in accurate placement in $94.5 \%$ compared to $91.4 \%$ in conventional screw placement. Duration of intraoperative radiation was significantly lower in robotic-guided compared to conventional procedures.

Postoperative administration of new opioid analgesics, duration of postoperative hospitalization and rates of adverse events seem to be lower in percutaneous roboticguided procedures compared to open robotic-guided and conventional procedures.

\section{Conflict of interest None.}

Open Access This article is distributed under the terms of the Creative Commons Attribution Noncommercial License which permits any noncommercial use, distribution, and reproduction in any medium, provided the original author(s) and source are credited.

\section{References}

1. Kantelhardt SR, Bock CH, Larsen J, Bockermann V, Schillinger W, Rohde V, Giese A (2009) Intraosseous ultrasound in the placement of pedicle screws in the lumbar spine. Spine 34(4):400-407

2. Wiesner L, Kothe R, Schulitz KP, Rüther W (2000) Clinical evaluation and computed tomography scan analysis of screw tracts after percutaneous insertion of pedicle screws in the lumbar spine. Spine 25(5):615-621

3. Schizas C, Michel J, Kosmopoulos V, Theumann N (2007) Computer tomography assessment of pedicle screw insertion in percutaneous posterior transpedicular stabilization. Eur Spine $\mathbf{J}$ 16(5):613-617

4. Pechlivanis I, Kiriyanthan G, Engelhardt M, Scholz M, Lücke S, Harders A, Schmieder K (2009) Percutaneous placement of pedicle screws in the lumbar spine using a bone mounted miniature robotic system: first experiences and accuracy of screw placement. Spine 34(4):392-398

5. Lonstein JE, Denis F, Perra JH, Pinto MR, Smith MD, Winter RB (1999) Complications associated with pedicle screws. J Bone Jt Surg (Am) 81(11):1519-1528

6. Güven O, Yalçin S, Karahan M, Sevinç TT (1994) Postoperative evaluation of transpedicular screws with computed tomography. Orthop Rev 23(6):511-516

7. Laine T, Lund T, Ylikoski M, Lohikoski J, Schlenzka D (2000) Accuracy of pedicle screw insertion with and without computer assistance: a randomised controlled clinical study in 100 consecutive patients. Eur Spine J 9(3):235-240

8. Wiesner L, Kothe R, Rüther W (1999) Anatomic evaluation of two different techniques for the percutaneous insertion of pedicle screws in the lumbar spine. Spine 24(15):1599-1603

9. Grass R, Biewener A, Dickopf A, Rammelt S, Heineck J, Zwipp H (2006) Percutaneous dorsal versus open instrumentation for fractures of the thoracolumbar border. A comparative, prospective study. Unfallchirurg 109(4):297-305

10. Foley KT, Gupta SK (2002) Percutaneous pedicle screw fixation of the lumbar spine: preliminary clinical results. J Neurosurg 97(1 Suppl):7-12

11. Kim JS, Choi WG, Lee SH (2010) Minimally invasive anterior lumbar interbody fusion followed by percutaneous pedicle screw fixation for isthmic spondylolisthesis: minimum 5-year follow-up. Spine J 10(5):404-409

12. Ringel F, Stoffel M, Stüer C, Meyer B (2006) Minimally invasive transmuscular pedicle screw fixation of the thoracic and lumbar spine. Neurosurgery 59(4 Suppl 2):361-366

13. Papadopoulos EC, Girardi FP, Sama A, Sandhu HS, Cammisa FP $\mathrm{Jr}$ (2005) Accuracy of single-time, multilevel registration in image-guided spinal surgery. Spine J 5(3):263-267

14. Schwarzenbach O, Berlemann U, Jost B, Visarius H, Arm E, Langlotz F, Nolte LP, Ozdoba C (1997) Accuracy of computerassisted pedicle screw placement. An in vivo computed tomography analysis. Spine 22(4):452-458

15. Kosmopoulos V, Schizas C (2007) Pedicle screw placement accuracy: a metaanalysis. Spine 32:111-120

16. Nakashima H, Sato K, Ando T, Inoh H, Nakamura H (2009) Comparison of the percutaneous screw placement precision of isocentric C-arm 3-dimensional fluoroscopy-navigated pedicle screw implantation and conventional fluoroscopy method with minimally invasive surgery. J Spinal Disord Tech 22(7): 468-472

17. von Jako RA, Carrino JA, Yonemura KS, Noda GA, Zhue W, Blaskiewicz D, Rajue M, Groszmann DE, Weber G (2009) Electromagnetic navigation for percutaneous guide-wire 
insertion: accuracy and efficiency compared to conventional fluoroscopic guidance. Neuroimage 47(Suppl 2):127-132

18. Mirza SK, Wiggins GC, Kuntz C 4th, York JE, Bellabarba C, Knonodi MA, Chapman JR, Shaffrey CI (2003) Accuracy of thoracic vertebral body screw placement using standard fluoroscopy, fluoroscopic image guidance, and computed tomographic image guidance: a cadaver study. Spine 28(4):402-413

19. Best NM, Sasso RC, Garrido BJ (2009) Computer-assisted spinal navigation using a percutaneous dynamic reference frame for posterior fusions of the lumbar spine. Am J Orthop 38(8): 387-391

20. Deininger MH, Unfried MI, Vougioukas VI, Hubbe U (2009) Minimally invasive dorsal percutaneous spondylodesis for the treatment of adult pyogenic spondylodiscitis. Acta Neurochir (Wien) 151(11):1451-1457

21. Arts MP, Nieborg A, Brand R, Peul WC (2007) Serum creatine phosphokinase as an indicator of muscle injury after various spinal and nonspinal surgical procedures. J Neurosurg 7:282-286
22. Kumbhare D, Parkinson W, Dunlop B (2008) Validity of serum creatine kinase as a measure of muscle injury produced by lumbar surgery. J Spinal Disord 21:49-54

23. Lehmann W, Ushmaev A, Ruecker A, Nuechtern J, Grossterlinden L, Begemann PG, Baeumer T, Rueger JM, Briem D (2008) Comparison of open versus percutaneous pedicle screw insertion in a sheep model. Eur Spine J 17(6):857-863

24. Regev GJ, Lee YP, Taylor WR, Garfin SR, Kim CW (2009) Nerve injury to the posterior rami medial branch during the insertion of pedicle screws: comparison of mini-open versus percutaneous pedicle screw insertion techniques. Spine 34(11):1239-1242

25. Huang QS, Chi YL, Wang XY, Mao FM, Lin Y, Ni WF, Xu HZ (2008) Comparative percutaneous with open pedicle screw fixation in the treatment of thoracolumbar burst fractures without neurological deficit. Zhonghua Wai Ke Za Zhi 46(2):112-114 